\title{
Tratamiento crónico combinado con iloprost y sildenafil en un paciente con hipertensión pulmonar primaria
}

\author{
Rodrigo Isa $\mathbf{P}^{1}$, Pablo Castro G ${ }^{1}$, Alejandro Martínez $\mathbf{S}^{1}$.
}

Aerosolized iloprost combined with a phosphodiesterase inhibitor in the treatment of primary pulmonary hypertension

Iloprost, a prostacyclin analogue administered by inhalation, improves hemodynamic and functional class variables in patients with primary pulmonary hypertension. However, repetitive inhalations are required due to its short term effects. One potential approach to prolong and increase the effects of aerosolized iloprost might be to combine its use with phosphodiesterase inhibitors. We report a 36 years old female patient with primary pulmonary hypertension treated with this combination. After 18 months of therapy the patient had an improvement in functional class and in the 6 min walk distance despite persistence of high pulmonary pressures. Our case is in agreement with the reported beneficial effect of the association of sildenafil and iloprost. We postulate that functional improvement in primary pulmonary hypertension is not always related to a decrease in pulmonary artery pressure (Rev Méd Chile 2004; 132: 353-6).

(Key Words: Iloprost; Phosphodiesterase inhibitors; Pulmonary heart disease; Sildenafil)

Recibido el 10 de octubre, 2003. Aceptado en versión corregida el 1 de diciembre, 2003.

${ }^{1}$ Departamento de Enfermedades Cardiovasculares, Hospital Clínico y Facultad de Medicina, Pontificia Universidad Católica de Chile.

$\mathrm{L}$ a hipertensión pulmonar primaria (HPP) es una enfermedad poco frecuente, caracterizada por un aumento de la presión en la arteria pulmonar, en ausencia de una causa originaria. Consiste en un compromiso de las arterias pulmonares precapilares, con hipertrofia de la media, fibrosis de la íntima, microtrombosis y lesiones

Correspondencia a: Dr. Pablo Castro G. Marcoleta 367, $6^{\circ}$ piso. Teléfono: 3543334. Fax: 6392037. E-mail: pcastro@med.puc.cl plexiformes $^{1}$. La mayoría de los individuos se presentan con disnea de esfuerzo, fatiga y síncope. La historia natural es hacia la falla cardíaca derecha y la muerte ${ }^{1,2}$.

El tratamiento de esta enfermedad ha sido desalentador. Sin embargo, se ha demostrado que la terapia con prostaciclina intravenosa (FLOLAN $^{\circledR}$ ) se asocia a una mayor sobrevida de los pacientes. Sin embargo, el alto costo limita su utilización ${ }^{3-5}$. 
Recientemente, estudios con derivados de la prostaciclina, con sistemas de administración menos complejos han mostrado resultados promisorios ${ }^{6-9}$. También, reportes aislados han mostrado una respuesta favorable con la administración de sildenafil, un inhibidor de la fosfodiesterasa tipo $5^{10,11}$.

Presentamos el caso de un paciente con HPP tratado con una combinación de un análogo de prostaciclina (iloprost inhalatorio) y sildenafil.

\section{Caso Cúnico}

Mujer de 36 años, sin antecedentes mórbidos, que inició en febrero de 2001 un cuadro de disnea de esfuerzo progresiva llegando a una capacidad funcional (CF) III. A los 3 meses de evolución consultó médico, indicándose propranolol en dosis de $10 \mathrm{mg}$ cada $8 \mathrm{~h}$. A las $48 \mathrm{~h}$ de iniciada la terapia, la paciente presentó un síncope en relación a esfuerzo, lo que motivó su hospitalización.

Examen físico: Presión arterial de 120/70 mmHg, frecuencia cardíaca de 70 lat/min, yugulares no ingurgitadas, palpación del latido ventricular derecho sostenido, primer ruido normal, aumento de la intensidad del componente pulmonar del segundo ruido, pulmones con murmullo vesicular normal, abdomen sin visceromegalias y extremidades sin edema.

Exámenes: Electrocardiograma en ritmo sinusal con bloqueo incompleto de rama derecha; la radiografía de tórax mostró crecimiento ventricular derecho y de la arteria pulmonar con oligoemia periférica; gases en sangre arterial, hemograma, velocidad de sedimentación, tiempo de protrombina y perfil bioquímico normales. Anticardiolipinas, anticoagulante lúpico, anticuerpos antinucleares, factor reumatoideo, anti ADN, $\mathrm{VIH}$, hepatitis B y $\mathrm{C}$ negativos. Un ecocardiograma mostró el ventrículo derecho dilatado e hipertrófico, insuficiencia tricuspídea leve con presión sistólica de arteria pulmonar (AP) de $90 \mathrm{mmHg}$, ventrículo izquierdo pequeño con septum paradojal y función sistólica normal. Angio TAC de tórax descartó tromboembolismo pulmonar.

Se realizó cateterismo cardíaco derecho que mostró presión de AP de 100/50 mmHg con presión de capilar pulmonar de $14 \mathrm{mmHg}$, débito cardíaco (DC) de 4,36 y resistencia vascular pulmonar (RVP) de 12,1 UW. Se administró adenosina IV en dosis hasta $125 \mu \mathrm{g} / \mathrm{min}$ sin cambios significativos de la presión de AP $(90 / 40 \mathrm{mmHg})$ ni del DC y RVP (10,1 UW). Se diagnosticó una HPP no reactiva. Test de marcha (TM) de $285 \mathrm{~m}$. Se inició tratamiento con neosintrón ${ }^{\circledR}$, manteniendo INR promedios de 2,5.

La paciente continuó con disnea, en CF III. Un ecocardiograma en febrero de 2002 mostró una presión sistólica de AP de $135 \mathrm{mmHg}$ y un DC estimado de 4,4 L/min. Se decidió iniciar sildenafil oral $50 \mathrm{mg}$ cada $6 \mathrm{~h}$, el cual fue bien tolerado, sin experimentar cambios significativos de la presión de AP (PAP) medida a los 30 min, 1, 2 y 3 h post sildenafil. Se controló TM que resultó en $375 \mathrm{~m}$, manteniéndose el tratamiento. A los cuatro meses de iniciada la terapia con sildenafil, la PAP persistió elevada. Se realizó una prueba con iloprost inhalatorio. La PAP estimada por ecografía fue de 120 $\mathrm{mmHg}$ posterior a la inhalación. Pese a no tener respuesta aguda, se decidió agregar iloprost cada 4 $\mathrm{h}$ a la terapia con sildenafil y anticoagulante orales.

Diez meses más tarde, en abril de 2002, la paciente se encontraba asintomática. Se realizó un ecocardiograma basal que reveló una PAPS de 137 $\mathrm{mmHg}$ con DC de 3,9 L/min y PA sistémica de $120 / 50 \mathrm{mmHg}$ y un control ecocardiográfico 60 min post iloprost inhalado que mostró ligera disminución de la PAPS a $111 \mathrm{mmHg}$ sin cambios en la PA sistémica y con ligero incremento del DC estimado (3,9 a 4,7 L). Se repitió TM que reveló una distancia recorrida de $490 \mathrm{~m}$.

Actualmente, a los 18 meses del inicio de la asociación de sildenafil e iloprost permanece en CF I.

\section{DisCUSIÓN}

El presente caso ilustra una respuesta clínica favorable con la combinación de un inhibidor de la fosfodiesterasa e iloprost en una paciente con HPP con síntomas avanzados y no reactiva a los test de reversibilidad.

En los pacientes con HPP no reactiva los bloqueadores de canales de calcio no son eficaces e incluso pueden ser deletéreos al producir 
hipotensión e isquemia ventricular derecha ${ }^{12-14}$; por esa razón no se utilizaron en nuestra paciente. Acorde a las recomendaciones de la literatura, el paso siguiente habría sido iniciar tratamiento con prostaciclina IV, la que ha demostrado ser útil en pacientes con síntomas avanzados y respuesta vasodilatadora aguda, pero también hasta en 30\% de los pacientes no reactivos, fenómeno que se explica por un remodelamiento a nivel de la microvasculatura y a una acción antiagregante plaquetaria ${ }^{3-5}$. Los pacientes tratados experimentan una mejoría de los síntomas, de la capacidad funcional y de la sobrevida. Sin embargo, esta terapia es de alto costo y de compleja administración lo que, sumado a la reticencia de la paciente al implante de un catéter central permanente y al riesgo de infecciones, impidió su utilización.

El óxido nítrico causa vasodilatación a través del aumento de los niveles de guanosina-monofosfato cíclico (GMPc) en la musculatura lisa vascular; sin embargo, su vida media es corta porque es degradado rápidamente por las fosfodiesterasas. La inhibición de una de ellas por sildenafil, un inhibidor específico de la fosfodiesterasa 5, disminuye la RVP en humanos con hipertensión pulmonar, en voluntarios sanos con vasoconstricción pulmonar hipóxica y en animales de experimentación ${ }^{10}$.

Michelakis y cols demostraron, en 13 pacientes en CF III y IV, referidos para trasplante pulmonar, que el sildenafil es superior al óxido nítrico en disminuir la PAP media e igualmente efectivo en disminuir selectivamente la RVP y, a diferencia de éste, el sildenafil produce un incremento significativo del índice cardíaco, lo que sugiere que no tendría un efecto inotropo negativo ${ }^{11}$.

En otro estudio, Wilkens y col evaluaron el efecto hemodinámico agudo de sildenafil, iloprost y la combinación en 5 pacientes con HPP en $\mathrm{CF}$ III y IV. El sildenafil redujo la PAP media y la RVP, el efecto de iloprost fue mayor y la combinación de iloprost con sildenafil tuvo un efecto aditivo y más prolongado sobre la PAP media que la administración de cada uno por separado $(13,8 \pm 1,4 \text { versus } 9,4 \pm 1,3 \mathrm{mmHg} ; \mathrm{p}<0,009)^{13}$. Esta asociación permite una administración más espaciada de iloprost ${ }^{13,14}$.

En otro estudio, Ghofrani y col, en 73 pacientes con hipertensión pulmonar de distintas etiologías y síntomas refractarios al iloprost, demostraron que la adición de sildenafil se asoció a una mejoría clínica $y$ en el TM $(256 \pm 30 \mathrm{~m}$ a $346 \pm 26 \mathrm{~m} \mathrm{p}<0,002)$, con beneficio sostenido a los doce meses de seguimiento ${ }^{14}$. En nuestra paciente no se demostró un efecto agudo del sildenafil sobre la PAP.

Se han desarrollado diferentes análogos de la prostaciclina para facilitar su administración, entre ellos se cuentan el iloprost (inhalatorio), el beraprost (oral) y el treprostinil (subcutáneo continuo). El beraprost, produjo una mejoría de la capacidad funcional en comparación a placebo en un seguimiento de 12 semanas $^{8}$. El treprostinil produjo una mejoría hemodinámica y en el TM; sin embargo $85 \%$ de los pacientes tuvo signos inflamatorios en el sitio de infusión ${ }^{9}$.

El iloprost inhalatorio es una terapia atractiva frente a otros análogos de la prostaciclina, por su efecto restringido al territorio pulmonar. Dentro de sus mecanismos de acción destaca un aumento en el clearence pulmonar de la endotelina-1, un potente vasoconstrictor y mitógeno de la musculatura lisa vascular que está sobreexpresado en el plasma y tejido pulmonar de pacientes con HPP ${ }^{15}$. Hoeper y cols observaron una mejoría en la capacidad de ejencicio con TM de 6 min (desde un basal de $278 \pm 96 \mathrm{~m}$ a $363 \pm 135 \mathrm{~m} \mathrm{p}<0,001$ ) y de variables hemodinámicas a los 12 meses de tratamiento ${ }^{6}$. Olschewski y cols en un estudio randomizado y controlado en 203 pacientes con HPP y CF III-IV mostraron, a los 12 meses de seguimiento, una mejoría de la $\mathrm{CF}$ y distancia caminada en el TM con iloprost en comparación a placebo $(16,8 \%$ vs $4,9 \%$ p $<0,007$ del punto final combinado) ${ }^{7}$.

Derivados de la prostaciclina, bloqueadores de la endotelina como bosentan ${ }^{16} \mathrm{y}$ en algunos casos los bloqueadores del calcio han mejorado la capacidad funcional y los parámetros hemodinámicos en pacientes con HPP 17,18. En pacientes con síntomas severos, la prostaciclina intravenosa prolonga su sobrevida ${ }^{3-5}$. Es interesante que en estos pacientes se ha observado una mejoría en la capacidad funcional y no necesariamente en sus cifras de PAP; esto pudiese significar que estos pacientes han experimentado un incremento en el gasto cardíaco y en el flujo pulmonar sin caídas en la PAP.

En el caso presentado, la terapia combinada de sildenafil e iloprost se asoció a una mejonía clínica 
objetivada en el TM, parámetro que se deteriora en la historia natural de los pacientes con HPP. No se objetivaron cambios hemodinámicos favorables en las PAP. Esta observación sugiere que no siempre

\section{REFERENCIAS}

1. Runo J, Loyd J. Primary Pulmonary Hypertension. Lancet 2003; 361: 1533-44.

2. D'alonzo GE, Barst RJ, Ayres SM, Bergofski E, Brundage B, Detre $K$ et al. Survival in patients with primary pulmonary hypertension: results from a national prospective registry. Ann Intern Med 1991; 115: 343-9.

3. Barst RJ, Rubin LJ, Long WA, McGoon M, Rich S, BADESCH D ET AL. A comparison of continuous intravenous epoprostenol (prostacyclin) with conventional therapy for primary pulmonary hypertension. N Engl J Med 1996; 334: 296-301.

4. McLaughun V, Shimngton A, Rich S. Survival in Primary Pulmonary Hypertension the Impact of Epoprostenol Therapy. Circulation 2002; 106: 1477-82.

5. Sitbon O, Humbert M, Nunes H, Parent F, García G, HeRve' PH ET AL. Long term intravenous epoprostenol infusion in primary pulmonary hypertension prognostic factors and survival. J Am Coll Cardiol 2002; 40: 780-8.

6. Hoeper MM, Schwarze M, EHLerding S, AdierSchuermeyer A, SpieKerkoetter E, Niedermeyer J et AL. Long term treatment of primary pulmonary hypertension with aerosolized iloprost, a prostacyclin analogue. N Engl J Med 2000; 342: 1866-70.

7. Olschewski H, Simonneau G, Galie N, Higenbottan T, Naeje R, Rubin L et aL. Inhaled iloprost is an effective treatment for severe pulmonary hypertension: a double-blind, placebo controlled, multicenter study. N Engl J Med 2002; 347: 322-9.

8. Galie N, Humbert M, Vachiery J, Vizza C, Kneussl M, MANES A ET AL. Effects of beraprost sodium, an oral prostacyclin analogue, in patients with pulmonary arterial hypertension: a randomized, double blind, placebo controlled trial. J Am Coll Cardiol 2002; 39: 1496-502.

9. Simonneau G, Barst RJ, Gale N, Naeje R, Rich S, Bourge $R$ et al. Continuous subcutaneous infusion of treprostinil, a prostacyclin analogue, in patients with pulmonary arterial hypertension: a double blind, randomized, placebo controlled trial. Am J Respir Crit Care Med 2002; 165: 800-4. existinía una estrecha correlación entre los efectos favorables sobre la capacidad funcional y la sobrevi$\mathrm{da}$, con la disminución de las cifras de PAP en pacientes tratados con prostaciclina y sus derivados.

10. Zhao L, Mason NA, Morrell NW, Kojonazarov B, SAdykov A, Maripov A et aL. Sildenafil inhibits hypoxia induced pulmonary hypertension. Circulation 2001; 104: 424-8.

11. Michelakis E, TymchaK W, Lien D, Webster L, HASHimoto K, ARcher S. Oral sildenafil is an effective and specific pulmonary vasodilator in patients with pulmonary arterial hypertension comparison with inhaled nitric oxide. Circulation 2002; 105: 2398-403.

12. Ricciardi MJ, Bossone E, Bach DS, Armstrong WF, RUBENFIRE M. Echocardiographic predictors of an adverse response to a nifedipine trial in primary pulmonary hypertension: diminished left ventricular size and leftward ventricular septal bowing. Chest 1999; 116: 1218-23.

13. Wilkens H, Guth A, König J, Forestier N, Cremers B, HenNEn B ET AL. Effect of inhaled iloprost plus oral sildenafil in patients with primary pulmonary hypertension. Circulation 2001; 104: 1218-22.

14. Ghofrani $H$, Rose F, Schermuly $R$, Olschewski $H$, WiedemanN R, KRECKel A Et al. Oral sildenafil as long term adjunct therapy to inhaled iloprost in severe pulmonary arterial hypertension. J Am Coll Cardiol 2003; 42: 158-64.

15. WilKens H, Bauer M, Forestier N, KöNIG J, Eichler A, SCHNEIDER S ET AL. Influence of inhaled iloprost on transpulmonary gradient of big endothelin in patients with pulmonary hypertension. Circulation 2003; 107: 1509-13.

16. Rubin LJ, Badesch DB, Barst RJ, Galie N, Black C, KeOCH A ET AL. Bosentan therapy for pulmonary arterial hypertension. N Engl J Med 2002; 346: 896-903.

17. Rich S, Kaufmann E, Levy PS. The effect of high doses of calcium channel blockers on survival in primary pulmonary hypertension. N Engl J Med 1992; 327: 76-81.

18. Rich S, BRundage BH. High dose calcium channel blocking therapy for primary pulmonary hypertension. Evidence for long term reduction in pulmonary arterial pressure and regression of right ventricular hypertrophy. Circulation 1987; 76: $135-41$ 